\title{
Dichotic and monotic interactions between speech and nonspeech sounds at different stimulus onset asynchronies
}

\author{
ROBERT J. PORTER, JR., and PAUL J. MIRABILE \\ University of New Orleans, New Orleans, Louisiana 70122 \\ and \\ Department of Otorhinolaryngology, Kresge Hearing Research Laboratory of the South \\ Louisiana State University Medical Center, New Orleans, Louisiana 70119
}

\begin{abstract}
Subjects' identification of stop-vowel "targets" was obtained under monotic and dichotic, forward and backward, masking conditions. Masks, or "challenges," were another stop-vowel or one of three nonspeech sounds similar to parts of a stop-vowel. Backward masking was greater than forward in dichotic conditions. Forward masking predominated monotically. Relative degree of masking for different challenges suggested that dichotic effects were predicated on interference with processing of a complex temporal array of auditory "features" of the targets, prior to phonetic decoding but subsequent to basic auditory analysis. Monotic effects seemed best interpreted as dependent on relative spectrum levels of nearly simultaneous portions of the two signals.
\end{abstract}

Dichotic presentation of certain speech sounds appears to result in at least two forms of interaction. One form, which we will refer to as phonetic competition, can be revealed by changes in subjects' performance which appear to be more a function of the phonetic message of the signals than of their acoustic structures (e.g., Studdert-Kennedy, Shankweiler, \& Pisoni, 1972). A second type of interaction, which we will refer to as central auditory masking, can be revealed by changes in subjects' performance which appear to be more a function of the acoustic properties of the competing signals than of their phonetic messages (e.g., Berlin, Porter, Lowe-Bell, Berlin, Thompson, \& Hughes, 1973). The present study deals primarily with this second type of interaction and extends the findings of the earlier work.

Berlin, Porter et al. (1973) asked subjects to identify speech-sound targets (stop-vowels) presented in dichotic competition with three types of speech and nonspeech masks, or "challenges." The dichotic challenge could be a steady-state vowel, a nonspeech two-formant "bleat" (which consisted of the second and third formants

This research was supported in part by National Institutes of Health, USPHS Grant NS-1 1647. Laboratory facilities were provided through a grant from The Kresge Foundation and by the Department of Psychology, University of New Orleans. We wish to thank Dr. F. S. Cooper and the Haskins Laboratories for use of the PCM and synthesis system; Pamela Weaver, Kathy Rich, Penny Bernard, and Gae Decker of the Kresge Lab staff for aid in manuscript preparation; and our colleagues, Chuck Berlin, Jack Cullen, Larry Hughes, and Jane Collins for helpful criticisms. A version of this paper was read by the second author at the $89 \mathrm{th}$ Meeting of the Acoustical Society of America, Austin, Texas, April 8-11, 1975. Please address correspondence to Robert J. Porter, Jr., Kresge Hearing Research Laboratory of the South, L.S.U. Medical Center, 1100 Florida Avenue, Building 164, New Orleans, Louisiana 70119. of a stop-vowel), or another stop-vowel. Performance on the target syllables was found to vary systematically with the challenge: greatest disruption was observed for stop-vowel challenges, next most by bleat challenges, and least by vowels. Since the bleats conveyed little or no phonetic information but did contain acoustic elements like those in the stop-vowels, this result suggested a central-masking effect, ${ }^{1}$ which was predicated more on the "acoustic similarity" of target and challenge than on their derived phonetic structure. The present study further explores the concept of "acoustic similarity" and its role in dichotic effects. Two new challenges, in addition to syllables and bleats, are used. One set of signals is used to explore the possibility that acoustic similarity may be defined primarily in terms of the presence of rapid transitions in the challenge. A second new challenge is included in order to determine if, alternatively, the acoustic similarity may be defined in terms of general spectral characteristics of the challenge. In addition, monotic and dichotic masking, under both asynchronous and synchronous presentations, are investigated.

\section{METHOD}

Twelve (12) right-handed, native speakers of American English (three males) served as paid subjects. All were students at the University of New Orleans and were selected for participation on the basis of: (1) normal hearing (re: ANSI, 1969) bilaterally at $250 \mathrm{~Hz}, 500 \mathrm{~Hz}, 1 \mathrm{kHz}, 2 \mathrm{kHz}$, and $4 \mathrm{kHz}$; and (2) ability to identify (with $86 \%$ or better accuracy) binaural presentations of the stop-vowels used. None of the subjects had participated in other speech or auditory perception experiments.

Subjects were asked to identify synthetic, three-formant, $250-\mathrm{msec}$, stop-consonant-vowel syllables (CVs) presented in dichotic and monotic competition with four types of synthetic challenges. All signals and tapes were produced on the 
Haskins Laboratories' parallel-formant synthesizer system. Sonagrams of the targets and challenges are presented in Figure 1. Each of the three target syllables, consisting of the stops $/ \mathrm{b}, \mathrm{d}, \mathrm{g} /$ plus a vowel approximating $/ \epsilon /$, are shown (in the order $/ b \epsilon, \mathrm{d} \epsilon, \mathrm{g} \epsilon /)$ at the top of Figure 1. The three CVs were presented in competition with one of the following challenges: (1) another of the stop-vowel syllables (" $\mathrm{CV}$ challenge"); (2) the isolated second formant of one of the other syllables (oneformant "bleats"); (3) one of three 60-msec, nonspeech, "sweep" signals, consisting of a linearly increasing, decreasing, or steady-state sweep of formants with frequencies symmetrically located around the center frequency of the F1, F2, and F3 of the stop-vowels; or (4) a "summed-speech mask" ("SS mask"), which was produced by arithmetically averaging the digitized waveforms of the three stop-vowel targets, thus producing a signal with the general spectral characteristics of a CV but lacking well-defined formant transitions.

Each of the three targets was paired with only two of the three challenges of a particular type. That is, a target syllable was never paired with itself, a bleat from itself, or with a sweep with transitions like those in the syllable. All targets were paired with the SS mask. These pairings yielded a basic set of six dichotic pairs for each type of challenge.

In addition to simultaneous competition, targets and their challenges were also presented at six onset asynchronies. Target onsets were made to either lead or lag challenge onsets by 20 , 40 , or $60 \mathrm{msec}$. Two 42 -trial randomizations (six pairs $\times$ seven asynchronies, including simultaneity) were constructed for each challenge condition.

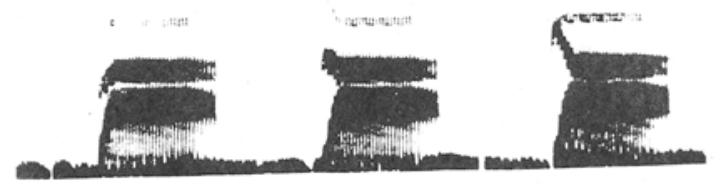

$B$
E
A
T
S

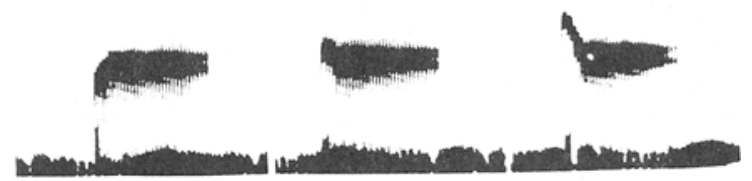

$S$
$W$
$E$
$E$
$P$
$S$
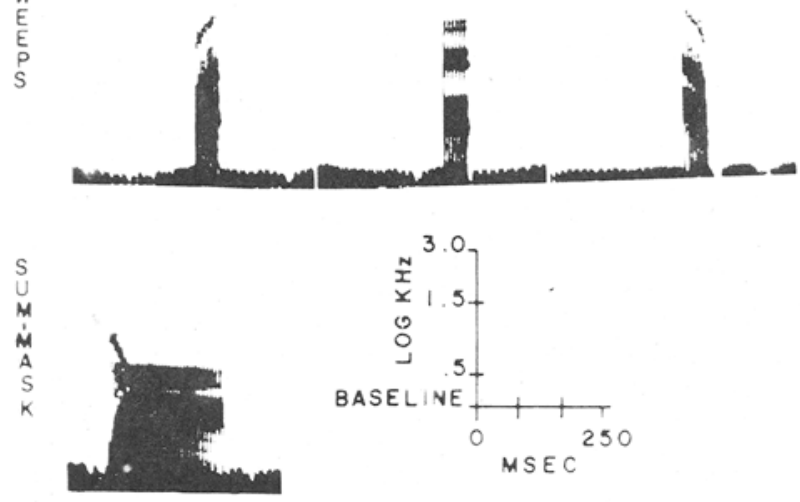

Figure 1. Sonagrams of targets and challenges.
In all dichotic conditions, the subjects were asked to identify target CVs in a designated ear and to ignore the challenge in the other ear (guessing at the $\mathrm{CV}$ if necessary). In all the monotic conditions, except monotic $\mathrm{CV}$ challenge, subjects were asked to identify the target $\mathrm{CV}$ which was electronically mixed with the challenge and presented to one ear. Since two CVs were presented together to a single ear in the monotic CV-challenge task, subjects were asked to identify both CVs in this condition, indicating which they were most sure of.

Subjects participated in two dichotic and two monotic listening sessions. Seven subjects received the dichotic listening condition first, the others the opposite order. Within either the monotic or dichotic session, half the subjects experienced the challenge conditions in the order: CV, bleat, SS mask, sweep. The other half were presented the conditions in the reverse order.

During each dichotic session, subjects experienced each challenge condition under each of two target-ear orientations twice; i.e., target $\mathrm{CV}$ left with the challenge right, and vice versa, twice each.

Within a challenge condition, dichotic or monotic, half the subjects first listened for targets in the right ear, then, in a second block of trials, for targets in the left ear. In the third and fourth blocks, targets were in the left, then right ear. The second half of the subjects experienced an opposite order of ear assignments. The two randomizations in each condition were presented in alternating order, within conditions, within a session.

Tapes were played on a Magnecord recorder (Model 1200), the output of which was amplified and presented via TDH-39 earphones.

All stimuli were presented at $65 \pm 1 \mathrm{~dB}$ SPL, as determined by equating the stimulus' sustained peak-to-peak voltages to that of a $1-\mathrm{kHz}$ tone that would generate $65 \mathrm{~dB}$ SPL through the earphones. Calibration checks were made periodically.

\section{RESULTS AND DISCUSSION}

Dichotic data were submitted to an analysis of variance, which revealed significant main effects of type of challenge, asynchrony (henceforth referred to as "lag"), and particular dichotic pairs $(p<.001)$. Of more interest, however, are the significant interactions of Challenge by Lag $(p<.01)$, Challenge by Pair $(p<.01)$, and Challenge by Pair by Lag $(p<.01){ }^{2}$ The significant Lag by Challenge interaction is shown in Figure 2. The ordinate presents the average percent-correct performance on the target stop-vowels. The parameter is the type of challenge, and the abscissa presents the six onset asynchronies, separated according to whether the target onset led or lagged the challenge onset. The abscissa is also labeled to reflect our view that the effects observed are due to either central forward, simultaneous, or backward auditory masking.

At simultaneity (zero onset-asynchrony), there is a systematically varying performance on the target $\mathrm{CV}$ as the type of challenge is varied. ${ }^{3}$ As in the Berlin, Porter et al. (1973) study, the amount of interference produced by a bleat is somewhat less than that produced by a CV challenge. Interestingly, the amount of interference produced by the SS mask is less than for either a bleat or a $\mathrm{CV}$, perhaps indicating that central disruption of the target requires that the challenge have some well-defined formant transition components. On the other hand, there is an absence of disruptive effects for the sweeps 


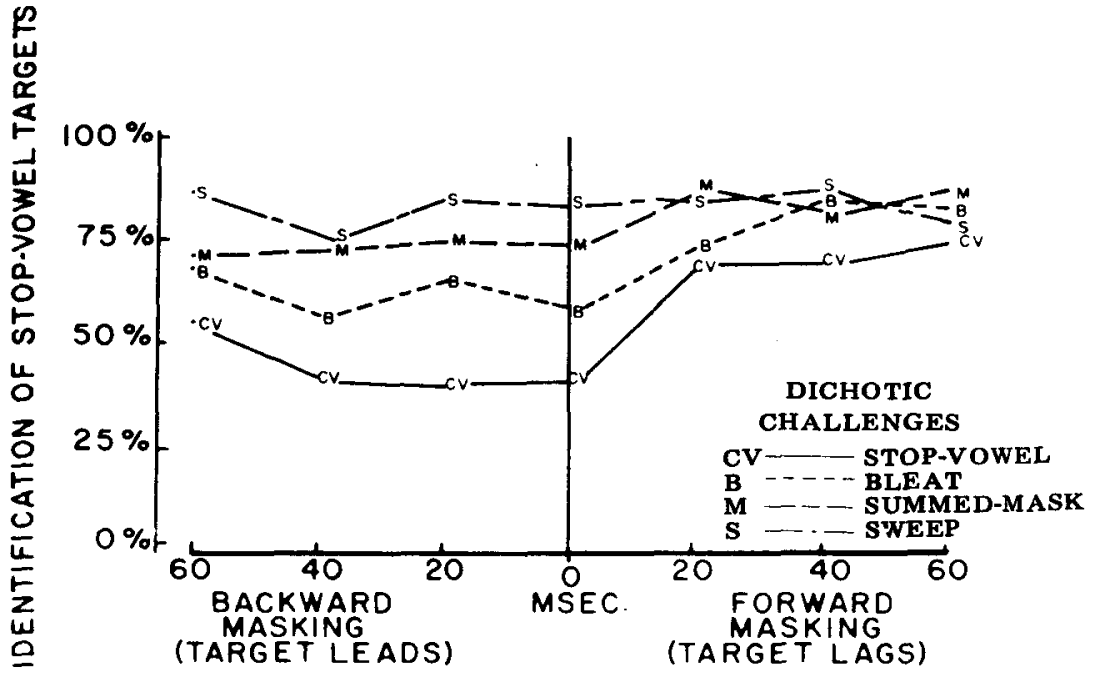

Figure 2. Dichotic results.

even though they do have well-defined transitions. It would appear that well-defined formant transitions may be necessary, but not sufficient, to produce central disruptive effects. It may be that only signals with both transitions and following steady states, like the bleats and CVs, can yield disruptive effects. These data thus suggest that the "acoustic similarity" underlying dichotic interaction is defined more in terms of complex features of the signals (e.g., transition + steady state) than in terms of simpler features like presence of transitions or presence of acoustic energy in certain frequency regions.

If targets are made to lead challenges, the relative disruptive effects observed at simultaneity are preserved, even up to $60-\mathrm{msec}$ asynchrony. That is, backward effects are like simultaneous effects: CVs give the biggest effects and the sweeps yield the least. Making the targets lag the challenges, however, results in a dramatic diminution or elimination of disruption with even the shorter asynchronies. The nearly equal backward and simultaneous performance coupled with the observation of greater backward than forward effects at comparable asynchronies jointly define what has been called the dichotic lag effect. This type of result was originally observed for dichotically presented stop-vowels by Studdert-Kennedy, Shankweiler, and Schulman (1970), and it has since been observed in a number of other studies (Berlin, Lowe-Bell, Cullen, Thompson, \& Loovis, 1973; Kirstein, 1971; Porter, 1971, 1974, 1975).

The interpretation of the lag effect has been the topic of some recent interest, with some investigators attributing it to phonetic competition of the signals, others to central auditory masking, and others to a combination of the two (discussions by Darwin, 1971; Kirstein, 1971). The present results would seem to favor an interpretation in terms of central auditory-masking effects, since the degree of lag effect (like the simultaneous effect) quite clearly varies with the acoustic similarity of the target and challenge. It is important to note, however, that more than central auditory masking is probably involved. For example, whereas the magnitude of backward masking varies systematically with the acoustic similarity of signals, the range of asynchronies over which these effects occur does not. This suggests the range of backward-masking effects may reflect something of the way in which the phonetic mechanism processes the auditory features. Porter (1974) for example, has suggested that the range of such backward masking may reflect the duration of storage and complex auditory features prior to the initiation of phonetic analysis. Present data support such an argument.

The forward-masking portion of the data may also provide information on the nature of phonetic processing. In Figure 1, the disruptive effects of the leading CV challenges are not eliminated by an offset of even $60 \mathrm{msec}$. We tentatively interpret this result as indicating a "precedence effect," that is, a tendency for a leading $\mathrm{CV}$ challenge to occasionally gain preferred access to the phonetic mechanisms. The range of this forward effect may give an indication of the duration of some aspects of phonetic analysis. Repp (1975), for example, drew such a conclusion on the basis of similar data.

The dichotic data are intriguing because they seem to provide some of the information concerning the central representation of a speech signal prior to phonetic analysis. That is, the bleats and $\mathrm{CV}$ challenges give larger effects than the SS mask does, presumably because of the presence of well-defined transitions, and also more than the sweeps do, presumably because bleats and CV challenges contain steady-state components. Under monotic conditions, in comparison, the signals might be expected to interact in terms of different aspects of the acoustic structure of the signals. There is some reason to suppose the monotic competition would result in different effects, since Studdert-Kennedy et al. (1970) 


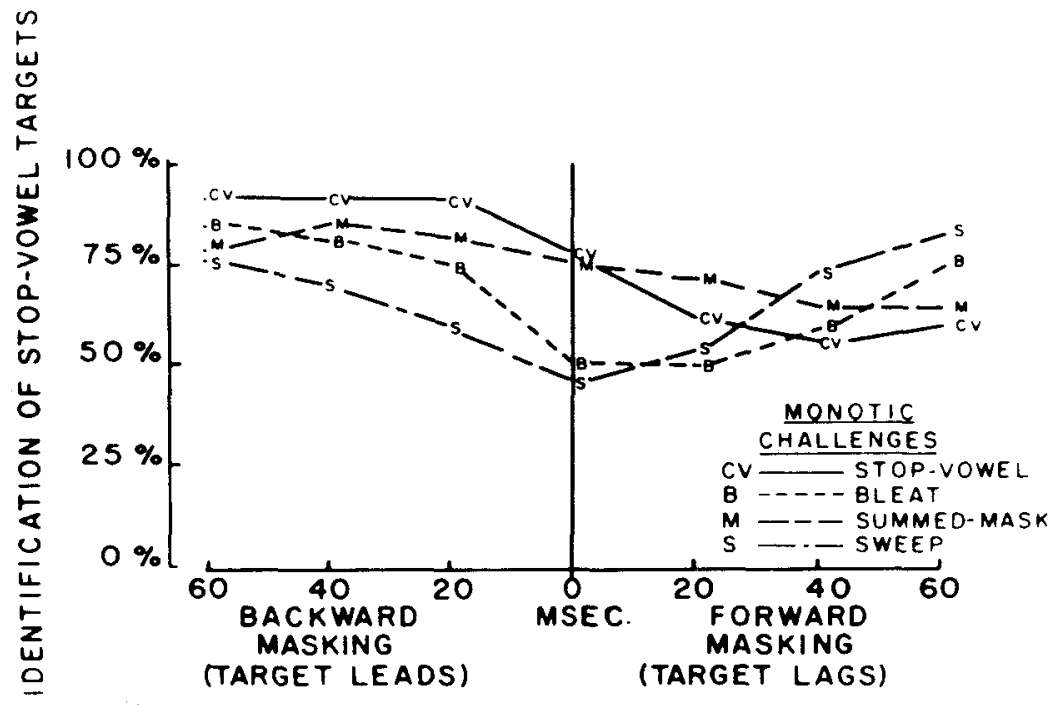

Figure 3. Monotic results.

have observed monotic presentation of asynchronous stop-vowels results in a perceptual advantage for the leading signal (see also Berlin, Lowe-Bell et al., 1973). This finding has been interpreted as reflecting, on the one hand, an "un-masking" of the leading signal as it is made to lead the lagging and, on the other, the simultaneous signal-to-noise-type masking of the lagging signal by the overlapping, trailing portion of the leading. Support for this interpretation has also been obtained in other studies (Porter, 1971; Repp, 1975).

Results of the monotic condition in the present study are presented in Figure 3. Analysis of variance for these data revealed significance for the main effects of lag $(p<.01)$ and challenge $(p<.05) .^{4}$

The monotic data are quite different from the dichotic. The sweep, which yielded little effect dichotically, tends to yield the most interference monotically. In addition, the overall masking effects for both the sweep and the bleat are somewhat greater than those for either the CV or the SS mask challenges. This we may attribute to the fact that while all signals were presented at $65 \mathrm{~dB}$ SPL overall, the acoustic energy is concentrated heavily in the second formant transition region for both the sweep and the bleat.

It is also apparent that for all of the signals, except the sweeps, forward-masking effects are larger than backward. In addition, although Challenge by Lag is nonsignificant $(p>.2)$, masking is more nearly symmetrical for the sweeps which lack the steady-state segments of the other signals. These results tend to support the conclusion that the monotic, simultaneous, and forwardmasking effects are, as Studdert-Kennedy et al. (1970) originally argued, principally a consequence of the unmasking of leading signals and the simultaneous masking of trailing CVs by the overlapping portions of the leading.
In summary, the monotic results are clearly different from those obtained dichotically, and a different set of acoustic aspects of the signals appears to be important in each case. Our interpretation is that the monotic effects are predicated primarily on the relative spectrum levels of nearly simultaneous portions of target and challenge and that the results reflect, for the most part, early auditory analysis. The relative magnitudes of monotic effects observed for each type of signal suggest, furthermore, that processes at this early level yield a representation of the signal in terms of features which are close to the signal's physical description in time and spectral energy. In the dichotic case, on the other hand, we conclude that the neural representation is at least one additional step away from the representation at the earlier stage. The relative magnitude of dichotic effects for different challenges suggests that the complex features represented at this central level bear quite an abstract relation to the acoustic parameters of the signal.

\section{REFERENCES}

Berlin, C. I., Berlin, H. L., Hughes, L., \& Dermody, P. Dichotic vs. monotic masking functions may reveal central organization for speech identification. Journal of the Acoustical Society of America, 1976, 59(1), S5. (Abstract)

Berlin, C. I., Lowe-Bell, S. S., Cullen, J. K., JR., Thompson, C. L., \& Loovis, C. F. Dichotic speech perception: An interpretation of right-ear advantage and temporal offset effects. Journal of the Acoustical Society of America, 1973, 53, 699.709.

BeruIN, C. I., \& MCNeIL, M. R. Dichotic listening. In N. J. Lass (Ed.), Contemporary issues in experimental phonetics. Springfield: Academic Press, 1976.

Berlin, C. I., Porter, R. J., JR., Lowe-Bell, S. S., Berlin, H. L., Thompson, C. L., \& Hughes, L. F. Dichotic signs of the recognition of speech elements in normals, temporal lobectomees, and hemispherectomees. IEEE Transactions of Audio Electroacoustics, 1973, AU-21(3), 189-195. 
Cullen, J. K., JR., Beruin, C. I., Hughes, L. F., Thompson, C. L. \& Samson, D. S. Speech information flow: A model. In Proceedings of a Symposium on Central Auditory Processing Disorders. Omaha: The University of Nebraska Medical Center, 1975. Pp. 108-127.

DARWIN. C. J. Dichotic backward masking of complex sounds. Quanerly Journal of Experimental Psychology, 1971, 23, 386-392.

Dorman, M. F., Raphael, L. J., Liberman, A. M., \& Repp, B. Maskinglike phenomena in speech perception. Journal of the Acoustical Society of America, 1975, 48. (Abstract) (Also in Haskins Laboratories Status Report on Speech Research, 1975, SR-24/43, 265-279.)

KirsteIn, E. F. Temporal factors in perception of dichotically presented stop consonants and vowels. Doctoral dissertation, University of Connecticut, 1971. Dissertation Abstracts, 1971, 32, 3035-B.

PoRTER. R. J. The effect of temporal overlap on the perception of dichotically and monotically presented CV syllables. Journal of the Acoustical Society of America, 1971, 50, 129. (Abstract)

Porter, R. J. The dichotic lag effect: Implications for the central processing of speech. Proceedings of Speech Communication Seminar, Stockholm, August 1-3, 1974. (To be published, New York: Wiley.)

Porter, R. J., JR. Effect of delayed channel on the perception of dichotically presented speech and nonspeech sounds. Journal of the Acoustical Society of A merica, 1975, 58, 884-892.

REPP. B. H. Dichotic forward and backward 'masking' between CV syllables. Journal of the Acoustical Society of America, 1975, 57, 483-496.

STUdDERT-KenNedy, M. Speech perception. In N. J. Lass (Ed.), Contemporary issues in experimental phonetics. Springfield: Thomas, 1975.

Studdert-Kennedy, M., Shankweiler, D., \& Pisoni, D. Auditory and phonetic processes in speech perception: Evidence from a dichotic study. Cognitive Psychology, 1972, 3, 455-466.

Studdert-Kennedy, M., Shankweiler, D., \& Schulman, S. Opposed effects of a delayed channel on perception of dichotically and monotically presented CV syllables. Journal of the Acoustical Society of America, 1970, 48, 599.602.

TURVEY, M. T. On peripheral and central processes in vision: Inferences from an information-processing analysis of masking with patterned stimuli. Psychological Review, 1973, 80, 1-52.

Whittaker, R. J., \& Porter, R. J., JR. Dichotic and monotic masking of CV's by second-formants with different transition starting values. Joumal of the Acoustical Society of America, 1976,60, S119.

\section{NOTES}

1. There is.some disagreement as to whether the term "masking" is appropriate here. Part of the difficulty seems to arise from the fact that the term can be used to refer to an experimental paradigm or, instead, to certain types of explanations of the results of such studies. In the paradigmatic sense, masking would seem to appropriately apply to any study in which perception of one signal ("target") is investigated as a function of the presence of another signal ("mask"). The use of the term in this way does not usually lead to debate. On the other hand, masking may be used to explain the mask-induced changes in target processing; in this sense, the term often refers to a presumed decrease in signal-to-noise ratio and a consequent decrease in detectability or intelligibility which occurs as a result of its being imbedded in the "noise" introduced by the presence of the mask. Limiting the explanatory definition of masking this way would seem counterproductive, particularly in view of results demonstrating various complex forms of interactions of masks and targets, which fail to lend themselves to explanations based strictly on signal-to-noise changes. Vision researchers have apparently been able to partially resolve the issue by using the term "masking" in a very general sense or by including a modifier which specifies the particular aspects of the process presumed to be taking place: for example, "pattern masking," "contrast masking," "central masking," "peripheral masking," etc. (e.g., Turvey, 1973). We, and others, have been following this approach to the use of the term in auditory studies (Dorman, Raphael, Liberman, \& Repp, 1975; Repp, 1975; Studdert-Kennedy, 1975). We ask the reader, therefore, to accept "central auditory masking" (occasionally referred to as "auditory masking") as referring to a particular type of central interaction, a model of which we develop in this paper and elsewhere (Cullen, Berlin, Hughes, Thompson, \& Samson, 1975; Porter, 1974).

2. The significance of the dichotic "pairs" and their interaction refers to the differences in effects depending upon a particular challenge competing with a particular target (e.g., /be/ vs. /ge/ and /ge/ vs. /de/ or /be/ vs. rising sweep and /ge/ vs. falling sweep, etc.). Unfortunately, this interesting set of interactions cannot be fully examined in these data, since not every target signal was presented against every challenge (e.g., $/ \mathrm{b} \epsilon /$ was never paired with a $/ \mathrm{b} \epsilon /$-bleat). Experiments presently in progress are examining these interactions in more detail in an effort to determine the spectral characteristics of effective challenges (Whittaker \& Porter, 1976).

3. Since, on half the trials, the-challenge was in the left ear and the target in the right, and vice versa, it is possible to analyze target performance, by ear, at simultaneity and each asynchrony. Such an analysis revealed a nonsignificant, but higher, performance on right-ear targets than on left-ear targets $(p>.05)$, and a nonsignificant Challesge by Lag by Ear interaction $(p>.05)$. There was, however, a significant Pair by Ear interaction $(p<.01)$, reflecting primarily the dependence of ear advantages on changes in overall performance under each challenge condition. There were no significant ear differences in the monotic conditions. The dichotic right-ear advantages are consistent with the findings of many dichotic CV studies in which right-ear advantages for signals like the targets used here are observed (Berlin \& McNeil, 1976). Since this right-ear advantage is not of particular relevance to the present investigation, it will not be considered further. A discussion of similar findings in this and other studies appears in Berlin, Berlin, Hughes, and Dermody (1976).

4. For purposes of data analysis, the monotic CV-challenge data were scored to yield a total-correct performance for lagging and leading members of the pairs. This total was then divided by 2. These data are, therefore, somewhat more susceptible to guessing inflation than in the case of the other conditions. Since the results parallel those previously observed, and since the proposed interpretation of the data does not appear to be compromised, no further transformation of these data were performed. For purposes of statistical analysis, only the monotic data for the three nonspeech challenges were included in the analysis of variance.

(Received for publication June 23, 1976; revision accepted February 2,1977.) 